\title{
Breeding by design of CMS lines on the platform of SSSL library in rice
}

\author{
Xin Luan • Ziju Dai • Weifeng Yang • Quanya Tan • \\ Qing Lu • Jie Guo • Haitao Zhu • Guifu Liu • \\ Shaokui Wang ( Guiquan Zhang
}

Received: 29 March 2019 / Accepted: 30 July 2019/Published online: 20 August 2019

(C) The Author(s) 2019

\begin{abstract}
Greatly increased yield by utilizing of heterosis has been a great success in rice over the past 40 years. The cytoplasmic male sterility (CMS) line is a key line of hybrid rice. However, the CMS line was very difficult to develop following the traditional breeding methods. Here, we report an example to develop and improve CMS lines sustainably by using the strategy of breeding by design under the platform of single segment substitution line (SSSL) library in the Huajingxian74 (HJX74) genetic background. The CMS lines HZA and HBA with the wild abortive (WA) cytoplasm from Zhenshan97A and BobaiA, respectively, were firstly developed in the HJX74 background through markerassisted selection. Then, four isonuclear alloplasmic CMS lines with the WA, dwarf wild abortive (DA), and Yegong abortive (YA) types of CMS cytoplasm, respectively, were developed by using the HZB maintainer line. According to the goal of design, E5-HXA and E5-HBA were developed by pyramiding five target genes, $W x^{g 1}, A L K, f g r, g s 3$, and OsMADS50, located on the chromosomal substituted segments in four SSSLs. The two CMS lines show the phenotypes of earlier in heading date, longer and narrower in grain shape, lower
\end{abstract}

X. Luan · Z. Dai · W. Yang - Q. Tan · Q. Lu · J. Guo • H. Zhu $\cdot$ G. Liu $\cdot$ S. Wang $(\bowtie) \cdot$ G. Zhang $(\bowtie)$

Guangdong Provincial Key Laboratory of Plant Molecular Breeding, State Key Laboratory for Conservation and Utilization of Subtropical Agro-Bioresources, South China Agricultural University, Guangzhou 510642, China

e-mail: shaokuiwang@scau.edu.cn

e-mail: gqzhang@scau.edu.cn in amylose content, and fragrant smell. Thus, the goal of design was achieved on the platform of SSSL library. Compared with that in the E5-HXA line carrying the $r f 3$ and $r f 4$ genes of XieqingzaoA, the CMS in the E5-HBA line with the $r f 3$ and $r f 4$ genes from BobaiA was susceptible to environmental impact and easy to be restored. Therefore, the balance between sterility and its restored ability in CMS lines should be concerned in breeding by design.

Keywords CMS · SSSL $\cdot$ Molecularbreeding $\cdot$ Breeding by design $\cdot$ Rice

\section{Introduction}

Rice (Oryza sativa) is the most important staple crop of the world and about half of the population in the world lives on it. Over the past decades, two tremendous leaps in enhancing rice yield have been achieved by the utilization of semi-dwarf gene in rice breeding (Spielmeyer et al. 2002), and by the utilization of heterosis in hybrid rice (Yuan 2017). The varieties of hybrid rice increased the grain yield by over $20 \%$ and had accounted for approximately $55 \%$ of the total rice planting area in China since the late 1980s (Cheng et al. 2007; Li et al. 2007).

Three-line system, which is composed by three different breeding lines: the cytoplasmic male sterility (CMS) line (A line), the maintainer line (B line), and the restorer line ( $\mathrm{R}$ line), is a main system in hybrid rice production. In the sporophytic CMS, the CMS lines 
with wild abortive (WA) cytoplasm, dwarf wild abortive (DA) cytoplasm, and Yegong abortive (YA) cytoplasm are commonly used in hybrid rice production ( $\mathrm{Li}$ et al. 2007; Dai et al. 2015). The CMS is restored by the fertility-restoring genes, $R f 3$ and $R f 4$. The $R f 3$ gene was mapped on chromosome 1 (Yao et al. 1997; Zhang et al. 1997), and the Rf4 gene was mapped on chromosome 10 (Yao et al. 1997; Zhang et al. 2002). Recently, the WA352 gene, which controls WA-CMS, and the Rf4 gene, which encodes a PPR protein, were cloned (Luo et al. 2013; Kazama and Toriyama 2014; Tang et al. 2014).

Peleman and van der Voort (2003) proposed the concept of "breeding by design." To conduct the strategy of breeding by design in rice, we have developed a library consisting of chromosomal single segment substitution lines (SSSLs) in rice by using Huajingxian74 (HJX74), an elite indica variety from South China, as the recipient, and 40 accessions from 7 species of AA genome in the Oryza genus as donors. To date, more than 2000 SSSLs have been developed (Zhang et al. 2004; He et al. 2005; Xi et al. 2006; He et al. 2017; Zhao et al. 2019). The genes for agronomic importance on the substituted segments in the SSSLs were analyzed. The gene information was collected including gene location, allelic variation, interaction of gene by gene and gene by environment, molecular function, and so on (Zeng et al. 2006; Zhao et al. 2007; Liu et al. 2008; Liu et al. 2010; Liu et al. 2012; Teng et al. 2012; Wang et al. 2012; Zhang et al. 2012; Chen et al. 2014; Qin et al. 2015; Wang et al. 2015; Yang et al. 2016; Zhao et al. 2016; Zhou et al. 2017; Yang et al. 2018; Zhu et al. 2018). To assess allelic variations at the $R f 3$ and $R f 4$ loci, the SSSLs carrying one of the loci in the substituted segments were selected from the library. Four alleles with a weak to strong restoration ability were identified at each locus (Cai et al. 2013). Based on the SSSL library, the platform of breeding by design for CMS lines was developed. Three isonuclear alloplasmic CMS lines with the HJX74 genetic background were developed (Dai et al. 2015). Then, we developed a platform for breeding by design of the CMS restorer lines based on the SSSL library. By using the strongest restorer alleles, $R f 3^{4}$ and $R f 4^{4}$, a restorer line with strong restoration ability was developed in the HJX74 genetic background (Dai et al. 2016).

In this study, we used the SSSL platform to develop new CMS lines to meet the needs of hybrid rice production. A set of new CMS lines with the WA, YA, and DA cytoplasm were developed. And then, two CMS lines with early heading and good quality were bred by design. The results indicate that the SSSL library is a powerful platform of breeding by design for CMS lines in rice.

\section{Materials and methods}

Plant materials

HJX74, an elite indica variety in South China, was used as the recipient of the SSSLs (He et al. 2005; Xi et al. 2006; He et al. 2017; Zhao et al. 2019). Four CMS lines used popularly in China, Zhenshan97A and BobaiA with WA cytoplasm, XieqingzaoA with DA cytoplasm, and HuanongA with YA cytoplasm, were used as the donors of abortive or sterile cytoplasm and recessive nuclear genes $r f 3$ and $r f 4$. The maintainer line HXB (also named H121B), which was previously developed from XieqingzaoB in the HJX74 genetic background (Dai et al. 2015), was used as the donor of rf3 and rf4 genes to develop new maintainer lines. Four SSSLs, W23-07-06-10-06 containing $W x^{g 1}$ and $A L K$ genes on the substituted segment of chromosome 6 from the Lemont, W15-05-09-06-08 with a substituted segment containing the $f g r$ fragrance gene on chromosome 8 from American Jasmine, W08-15-08-28 with the gs3 gene on the substituted segment of chromosome 3 from IR64, and W23-03-08-09-27-82 with OsMADS50 gene on the substituted segment of chromosome 3 from Lemont, were used as donors of the target genes (Table 1).

All of the plant materials used in this study were planted in two cropping seasons each year, in the experimental station at South China Agricultural University, Guangzhou $\left(23^{\circ} 07^{\prime} \mathrm{N}, 113^{\circ} 15^{\prime} \mathrm{E}\right)$, China, from 2012 to 2017. The spring cropping season was from late February to middle July, and the fall cropping season was from late July to middle November. Field management, including irrigation, fertilizer application, and pest control, was kept according to the recommendation of the area.

\section{Development of breeding lines}

To develop maintainer lines in the HJX74 genetic background, HJX74 was crossed with BobaiB and Zhenshan97B to produce $F_{1}$ hybrids, then $F_{1}$ hybrids 
Table. 1 Chromosomal substituted segments with target genes in the SSSLs used in this study

\begin{tabular}{|c|c|c|c|c|c|}
\hline Donor & Chr. & Substitution segment & $\begin{array}{l}\text { Interval of substituted segments } \\
(\mathrm{cM})\end{array}$ & Target gene & $\begin{array}{l}\text { Target } \\
\text { trait }\end{array}$ \\
\hline Lemont & 6 & $\begin{array}{l}\text { RM508-RM190 }\left(W x^{g l}\right) \\
\text {-RM225-RM253-RM402-ALK- } \\
\text { RM539-PSM388-RM136-RM3-RM541 }\end{array}$ & $1.4-68.5$ & $\begin{array}{l}W x^{g l} \\
A L K\end{array}$ & $\begin{array}{l}\text { MAAC } \\
\text { HGT }\end{array}$ \\
\hline $\begin{array}{l}\text { American } \\
\text { Jasmine }\end{array}$ & 8 & RM515-PSM522-fgr-PSM525-RM223 & $72.1-75.7$ & $f g r$ & Fragrance \\
\hline IR64 & 3 & PSM485-gs3-RM6146-RM3646-RM16 & $83-87.9$ & $g s 3$ & LG \\
\hline Lemont & 3 & RM306-RM3202-RM3894-RM523 & $1.1-5.5$ & OsMADS50 & $\mathrm{EH}$ \\
\hline
\end{tabular}

$M A A C$, medium apparent amylose content; $H G T$, high gelatinization temperature; $L G$, long grain; $E H$, early heading

were backcrossed with $\mathrm{HJX} 74$ to produce $\mathrm{BC}_{1} \mathrm{~F}_{1}$, respectively. A total of 228 polymorphic SSR markers, including RM1/RM10340 linked to rf3 and RM5373/ RM6100 linked to $r f 4$, were selected on the rice microsatellite maps (Chen et al. 1997; McCouch et al. 2002; Zhang et al. 2007), to detect target genes $r f 3$ and $r f 4$ and genetic background in every generation by markerassisted selection (MAS). After successive backcrossing, two new maintainer lines, HBB developed from BobaiB and HZB from Zhenshan97B, were developed in $\mathrm{BC}_{5} \mathrm{~F}_{2}$ generations.

To develop a four-segment pyramiding line, W2307-06-10-06 with the $W x^{g 1}$ and ALK genes, W15-05-0906-08 with the $f g r$ gene, W08-15-08-28 with the $g s 3$ gene, and W23-03-08-09-27-82 with the OsMADS50 gene in their substituted chromosomal segments were selected from the HJX74-SSSL library (Table 1). Two double segment substitution lines (DSSLs) were developed from the crosses of W23-07-06-10-06/W15-0509-06-08 and W08-15-08-28/W23-03-08-09-27-82, respectively. Then, the two DSSLs were crossed to develop a four-segment pyramiding line by MAS.

To develop early heading and good quality CMS lines, HXB and HBB were respectively crossed with the four-segment pyramiding line. Then, the $F_{1}$ hybrids were backcrossed with the four-segment pyramiding line. Finally, two new maintainer lines, E5-HXB developed from $\mathrm{HXB}$ and $\mathrm{E} 5-\mathrm{HBB}$ from $\mathrm{HBB}$, were respectively obtained in $\mathrm{BC}_{1} \mathrm{~F}_{2}$ generations by MAS.

Examination of pollen and spikelet fertility

To examine pollen fertility, 6-9 mature flowers were collected from the upper one-third of the panicles of plants during the flowering time. The pollens were stained with $1 \% \mathrm{I}_{2}$-KI solution containing $0.1 \%(w / v)$ iodine and $1 \%(w / v)$ potassium iodide. More than 300 pollen grains were randomly counted per plant under an optical microscope. The numbers of stainable pollens and un-stainable pollens in each individual were recorded. To observe spikelet fertility, three panicles per plant were harvested during the maturation time. Ten to twenty plants were recorded for each variation.

Investigation of traits and statistical analysis

Each of the traits was tested in 10-20 plants per line during the fall cropping season in 2012-2017. The apparent amylose content (AAC) and gelatinization temperature (GT), which was indirectly estimated via alkali spreading value (ASV), were tested following procedures described in previous studies (Teng et al. 2012; Teng et al. 2013). Leaves and dehulled rice were used to evaluate the fragrance. To test fragrance using leaves, one or two leaves per line were cut into small pieces and put into $15-\mathrm{mL}$ tubes with $10 \mathrm{~mL}$ of $1.7 \%$ $\mathrm{KOH}$. After $30 \mathrm{~min}$, the boxes were opened and immediately smelled (Sood and Siddiq 1978). To test fragrance using dehulled rice grains, 10 seeds from each line were chewed individually. Rice materials were then scored as fragrant or non-fragrant. Statistical analyses of the tested traits were processed by using Microsoft Excel 2010 and SPSS version 18.0.

\section{Results}

Development of new maintainer lines, HZB and HBB, in the HJX74 genetic background

To develop two new maintainer lines in the HJX74 genetic background, HJX74 was crossed with 
a

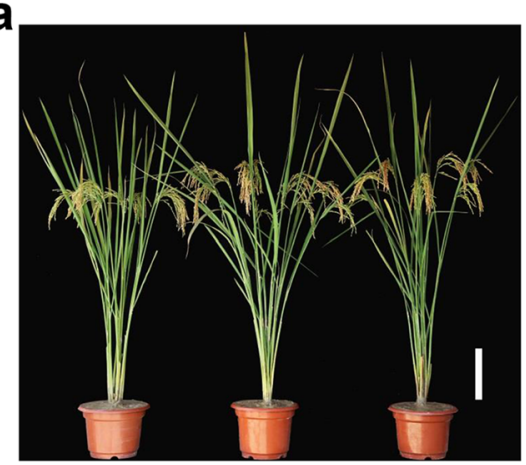

HJX74

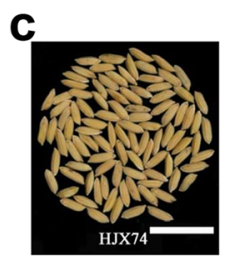
HZB

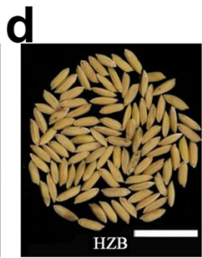
HBB

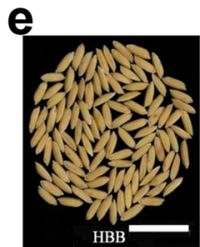

Fig. 1 Genotypes and phenotypes of HZB and HBB. a Plant morphology of HJX74, HZB, and HBB. Scale bar, $15 \mathrm{~cm}$. b Position of the target substituted segments in the genome of HZB and HBB. Vertical bars are a graphical representation of chromosomes. Black parts are substituted segments from Zhenshan97B/BobaiB with target genes, and white parts are

maintainer lines Zhenshan97B and BobaiB to produce $\mathrm{F}_{1}$ hybrids, respectively. Then, five times successive marker-facilitated backcrossing with HJX74 was performed to develop $\mathrm{BC}_{5} \mathrm{~F}_{1}$ populations. Two new HJX74 maintainer lines, HZB and HBB, with the rf3 and $r f 4$ genes derived from Zhenshan97B and BobaiB, were bred in the $\mathrm{BC}_{5} \mathrm{~F}_{2}$ generation, respectively (Fig. 1a). In the HZB line, only two chromosomal substituted segments from Zhenshan97B were detected in the HJX74 genetic background, a 39.1-cM-length segment containing the $r f 3$ locus on chromosome 1 and a 3.6-cM-length segment containing the $r f 4$ locus on chromosome 10. Similarly, the HBB line carries a 27.6-cM-length segment with $r f 3$ locus on chromosome 1 and a 39.9-cM-length segment with rf4 locus on chromosome 10 from BobaiB in the HJX74 genetic background (Fig. 1b).

Because only two chromosomal segments were substituted in HZB and HBB lines compared with HJX74, no significant differences of agronomic traits, such as grain shape, panicle number per plant, plant height, and heading date, were detected
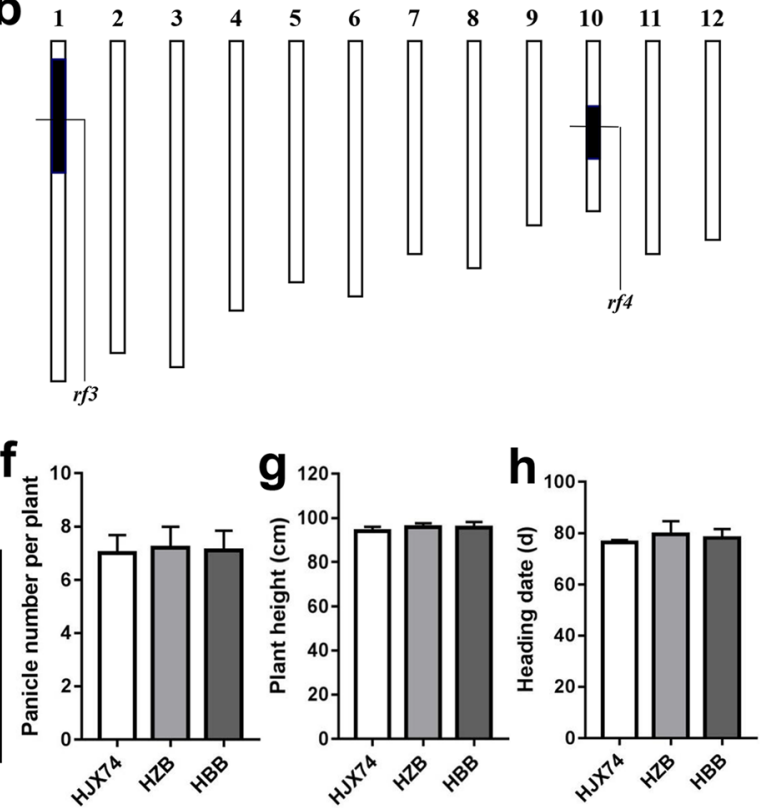

genetic background from HJX74. c-e Grain shapes of HJX74, HZB, and HBB. Scale bar, $2 \mathrm{~cm}$. $\mathbf{f}$ The number of panicles per plant of HJX74, HZB, and HBB. g Plant height of HJX74, HZB, and HBB. h Heading date of HJX74, HZB, and HBB. Error bars represent $\mathrm{SD}$

between the maintainer lines (HZB and HBB) and HJX74 (Fig. 1c-h).

Development of isonuclear alloplasmic CMS lines of HZB

Using the maintainer line HZB as the nuclear donor, four isonuclear alloplasmic CMS lines, WA-HZA1, WA-HZA2, DA-HZA, and YA-HZA, were developed (Fig. 2a). To develop the isonuclear alloplasmic CMS lines, four CMS lines, Zhenshan97A and BobaiA with WA cytoplasm, XieqingzaoA with DA cytoplasm, and HuanongA with YA cytoplasm, were used as cytoplasm donors to cross with HZB. After successive backcrossing for six times, the pollens of all $\mathrm{BC}_{6} \mathrm{~F}_{1}$ plants were completely sterile in the four populations. The anthers were white and slender, and the pollen grains were shrinking and un-stained with $1 \% \mathrm{I}_{2}-\mathrm{KI}$ solution (Fig. 2d-g). As control, the anthers and pollen grains in maintainer line HZB were normal (Fig. 2h). In the following successive backcrossing, the sterility was complete and steady with $100 \%$ of abortive pollens from 

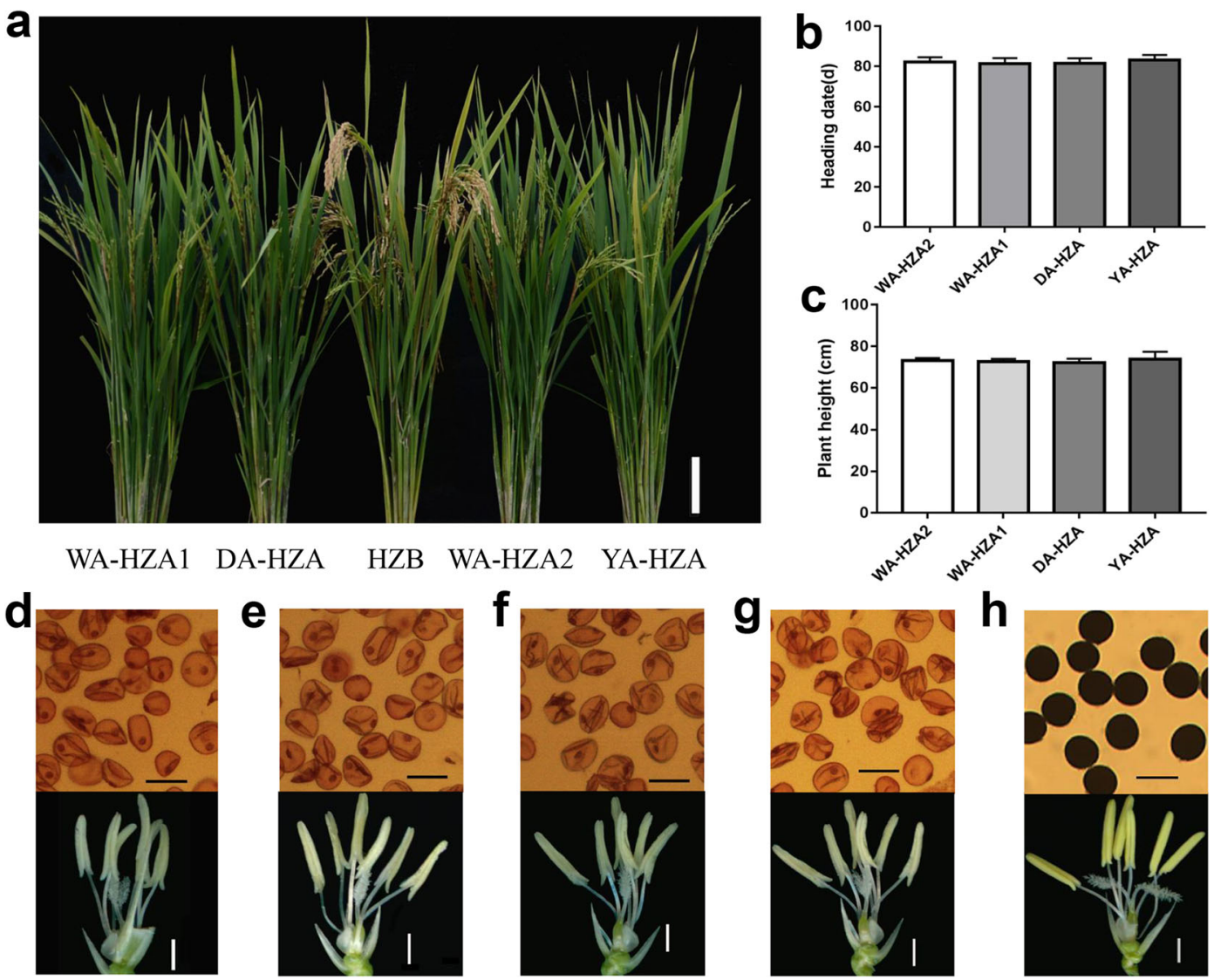

Fig. 2 Phenotypes of four isonuclear alloplasmic CMS lines. a Plant morphology of WA-HZA1, DA-HZA, HZB, WA-HZA2, and YA-HZA. The cytoplasm of WA-HZA1, DA-HZA, WAHZA2, and YA-HZA was derived from BobaiA, XieqingzaoA, Zhenshan97A, and HuanongA, respectively. Scale bar, $15 \mathrm{~cm}$. b, c

generation to generation in the four populations. In consequence, four isonuclear alloplasmic CMS lines, WA-HZA1, WA-HZA2, DA-HZA, and YA-HZA, in the HJX74 genetic background were developed.

The traits of isonuclear alloplasmic CMS lines were tested and compared with each other. The plant heights were in $72.2-73.9 \mathrm{~cm}$ and the heading dates were $81.3-$ 83.1 days with no significant difference among four CMS lines (Fig. 2b, c). These results indicated that the cytoplasm from WA, DA, and YA did not cause difference in the traits among the isonuclear alloplasmic CMS lines.

Improvement of the CMS maintainer lines of HBB and HXB

To conduct breeding by design of CMS lines with early heading and good quality, four SSSLs with the target
Heading date and plant height of four isonuclear alloplasmic CMS lines. Error bars represent SD. $\mathbf{d}-\mathbf{h}$ Morphology of anthers and pollen grains of WA-HZA1, DA-HZA, WA-HZA2, YA-HZA, and $\mathrm{HZB}$, respectively. Pollen grains were stained with $1 \% \mathrm{I}_{2}-\mathrm{KI}$; Scale bars, $1 \mathrm{~mm}$ (anthers); $50 \mu \mathrm{m}$ (pollen)

genes $W x^{g l}$ and $A L K$ from Lemont, $f g r$ from American Jasmine, gs3 from IR64, and OsMADS50 gene from Lemont were selected from the SSSL library to develop pyramiding line with those loci (Fig. 3; Table 1). At first, two DSSLs were respectively developed from the crosses of W23-07-06-10-06/W15-05-09-06-08 and W08-15-08-28/W23-03-08-09-27-82. And then, a line with the four substituted segments, named E5, was developed from the cross of the two DSSLs by MAS (Fig. 3). Using the maintainer line HBB developed in this study, and the maintainer line HXB (H121B) developed previously in our lab (Dai et al. 2015) as the donors of $r f 3$ and $r f 4$, two new maintainer lines, E5-HBB and E5-HXB, were respectively developed from the crosses between the maintainer lines (HBB and HXB) and E5 by MAS (Fig. 4a).

The traits controlled by the target genes in the E5$\mathrm{HBB}$ and E5-HXB were investigated to evaluate the 

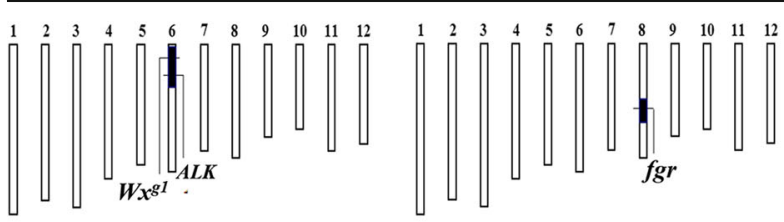

W23-07-06-10-06

W15-05-09-06-08

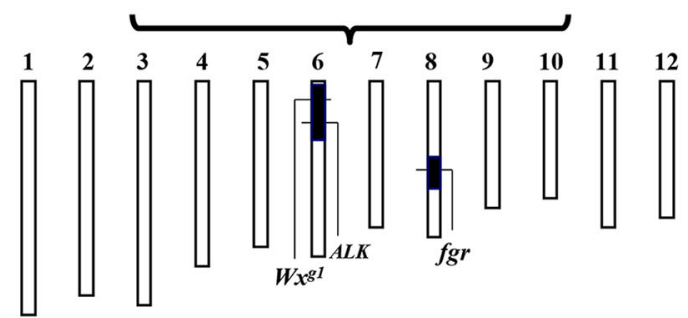

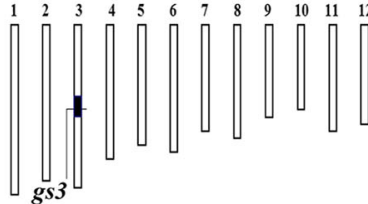

W08-15-08-28
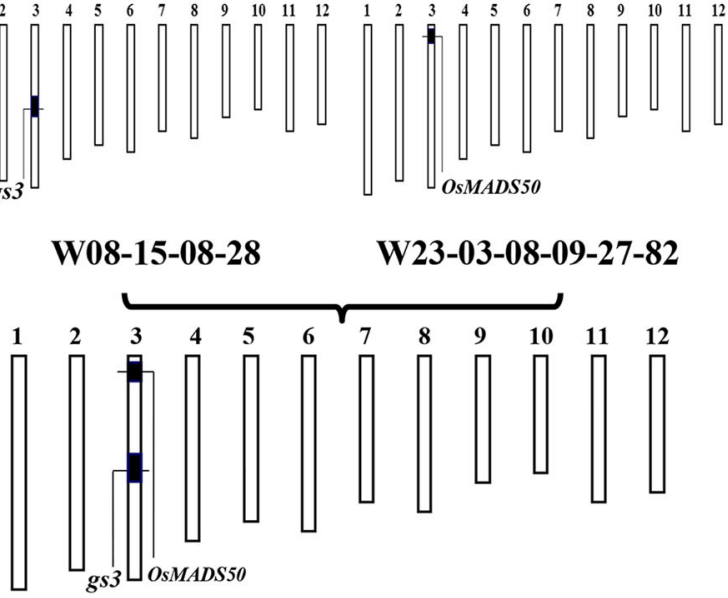

W23-03-08-09-27-82
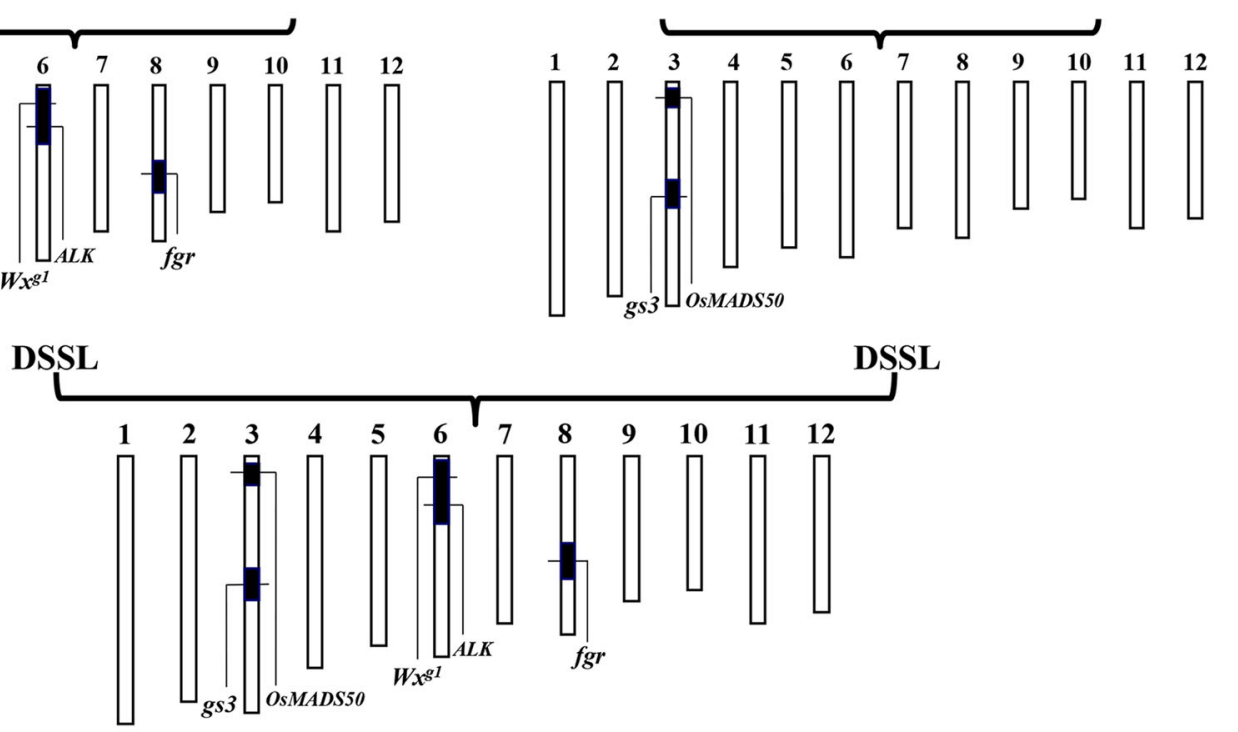

\section{.}




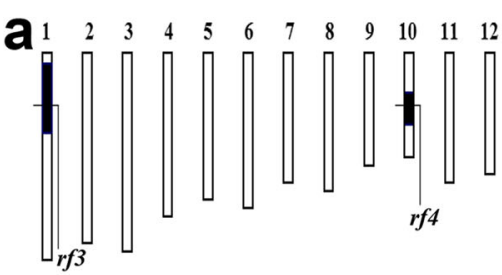

HBB

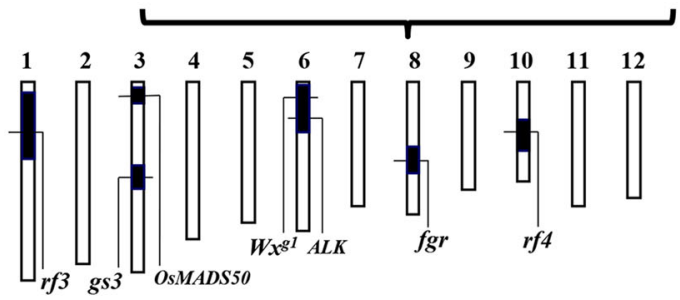

E5-HBB
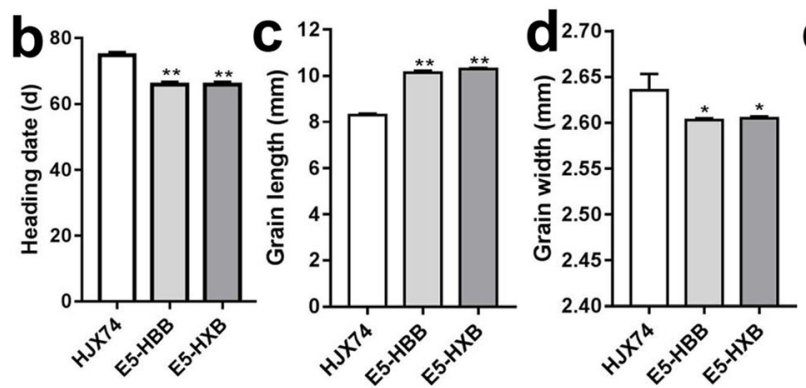

Fig. 4 Development of the E5-HBB and E5-HXB maintainer lines. a Development schemes of the E5-HBB and E5-HXB maintainer lines based on the HJX74 SSSL library. E5-HBB was developed from the cross of HBB and E5 (a four-segment pyramiding line). E5-HXB was developed from the cross of HXB and E5 in the same way. The vertical bars are a graphical representation of the chromosomes. Black regions represent

Jul) (Fig. 5e, f; Table 2). In spring cropping seasons, the pollen grains of E5-HBA presented spherical abortion type, stained abortion type, and even some fertility (Fig. 5e; Table 2). By contrast, typical abortion type of pollen grains was always observed in fall cropping seasons of E5-HBA (Fig. 5f). These results indicated that the CMS in the E5-HBA line was more susceptible to environmental factors compared with that in other CMS lines.

To evaluate the restored ability of CMS lines, two restorer lines developed in our lab, P46 and Rnn, were test-crossed with the E5-HBA and E5-HXA in the fall cropping season of 2017, respectively. The higher degree of restoration was detected in E5-HBA in terms of pollen fertility than that in E5-HXA when test-crossed with P46 and Rnn (Fig. 5g, i). The pollen fertility in the $F_{1}$ hybrids

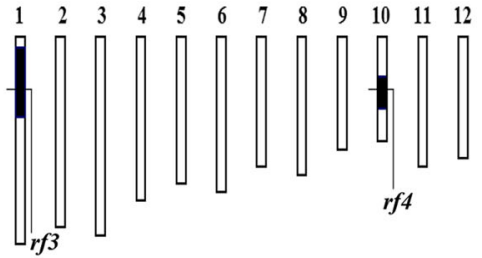

E5
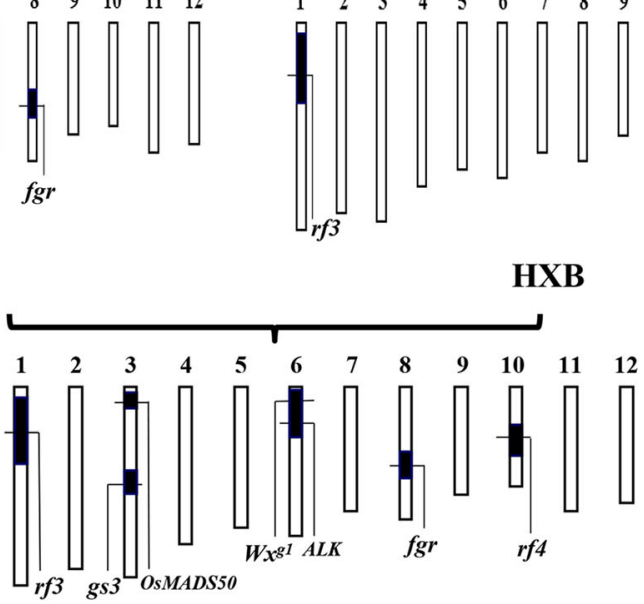

E5-HXB

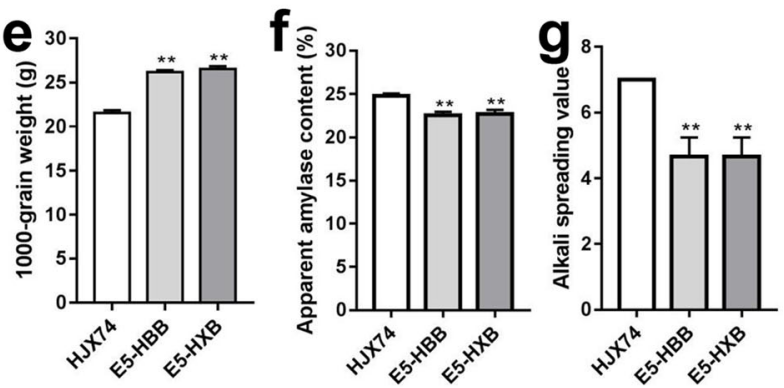

substitute segments with target genes, and white regions represent the HJX74 genetic background. b Heading date. c Grain length. d Grain width. e 1000-grain weight. f Apparent amylase content. g Alkali spreading value. Error bars represent SD. Single asterisk (*) and double asterisks $(* *)$ represent significance at the 0.05 and 0.01 probability level by $t$ test

from CMS line E5-HBA (95.6\% in P46 and 95.7\% in Rnn) was significantly higher than that from CMS line E5HXA (90.6\% in P46 and 92.7\% in Rnn) (Fig. 5g, i) although the difference was not found in the spikelet fertility (Fig. 5h, j). These results indicated that the CMS in the E5HBA line was easily restored than that in the E5-HXA line.

The pollen sterility of E5-HBA was influenced by environment

The pollen sterility of E5-HBA was incomplete and unsteady in the spring cropping season in 2013-2017 (Table 2). To test the period from incomplete sterility to complete sterility, the E5-HBA CMS line was sowed in 6 times, from Mar 2 to Jul 13 in 2015. The pollen fertility at the 6 sowing times was $5.03-5.07 \%, 1.11-$ $1.42 \%, 0.65 \%, 0-0.12 \%, 0$, and 0 in E5-HBA CMS 

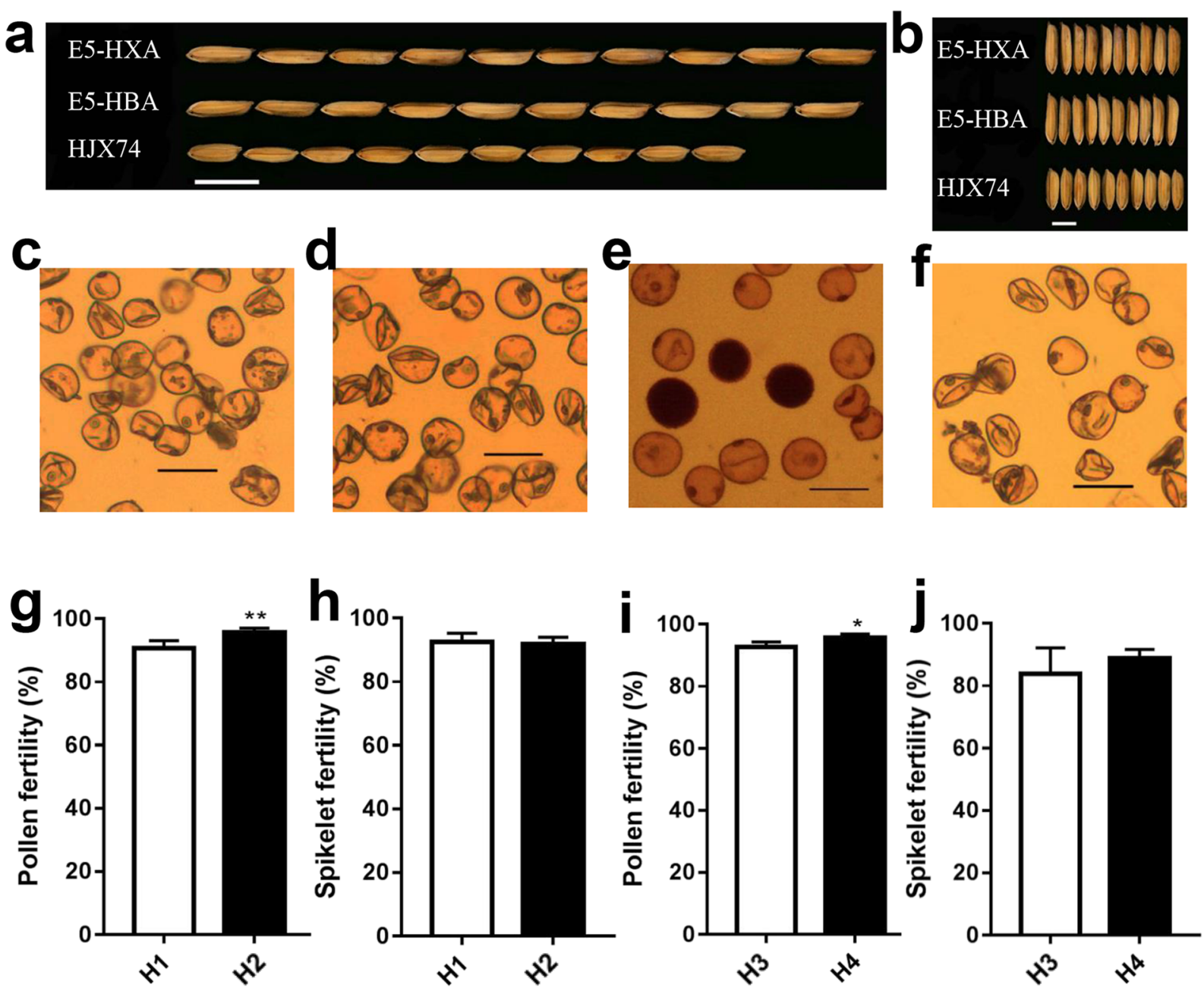

Fig. 5 Phenotypes of E5-HXA and E5-HBA. a, b Grain shapes of E5-HXA and E5-HBA. Scale bar, $1 \mathrm{~cm}$ (a); $0.5 \mathrm{~cm}$ (b). c, d Morphology of pollen grains of E5-HXA in spring cropping season (c) and fall cropping season (d). e, f Morphology of pollen grains of E5-HBA in spring cropping season (e) and fall cropping season (f). Pollen grains were stained with $1 \% \mathrm{I}_{2}-\mathrm{KI}$; Scale bar, $50 \mu \mathrm{m}$. g Pollen fertility of the $\mathrm{F}_{1}$ hybrids from the crosses between E5-HBA, E5-HXA, and restorer line P46. h Spikelet

fertility of the $F_{1}$ hybrids from the crosses between E5-HBA, E5-HXA, and restorer line P46. i Pollen fertility of the $\mathrm{F}_{1}$ hybrids from the crosses between E5-HBA, E5-HXA, and restorer line Rnn. j Spikelet fertility of the $F_{1}$ hybrids from the crosses between E5-HBA, E5-HXA, and restorer line Rnn. (H1), E5-HXA/P46; (H2), E5-HBA/P46; (H3), E5-HXA/Rnn; (H4), E5-HBA/Rnn. Error bars represent the $\mathrm{SD}, n=12$

Table 2 Pollen fertility of E5-HBA and E5-HXA in 9 crop seasons

\begin{tabular}{|c|c|c|c|c|c|c|c|c|c|}
\hline \multirow[t]{2}{*}{ CMS } & \multicolumn{9}{|c|}{ Pollen fertility $(\%)$} \\
\hline & 2013 fall & 2014 spring & 2014 fall & 2015 spring & 2015 fall & 2016 spring & 2016 fall & 2017 spring & 2017 fall \\
\hline E5-HXA1 & 0 & 0 & 0 & 0 & 0 & 0 & 0 & 0 & 0 \\
\hline E5-HXA2 & 0 & 0 & 0 & 0 & 0 & 0 & 0 & 0 & 0 \\
\hline E5-HBA1 & 0 & $24.09 \pm 5.91$ & 0 & $8.6 \pm 4.68$ & 0 & $0.92 \pm 0.42$ & 0 & $3.14 \pm 1.51$ & 0 \\
\hline E5-HBA2 & 0 & $16.89 \pm 6.36$ & 0 & $11.3 \pm 2.65$ & 0 & $1.25 \pm 1.01$ & 0 & - & 0 \\
\hline
\end{tabular}

Data shown are mean $\pm \mathrm{SE}, n=10$ 
line, and was, as control, 0 in all E5-HXA CMS lines (Table 3). The result indicated that the pollen sterility in E5-HBA was incomplete and unsteady when seed is sown before June 1, while complete and steady when seed is sown after June 1.

\section{Discussion}

Breeding by design, which can be achieved by three key procedures: mapping loci involved in all agronomic relevant traits; assessment of the allelic variation at those loci; and breeding by design of superior genotypes comprising a combination of favorable alleles at all loci, was proposed by Peleman and van der Voort (2003). We have been working on breeding by design in rice following a strategy of the three steps more than 20 years. At the first step, we developed a library of single segment substituted lines (SSSLs) in rice by the using HJX74 as the recipient and 40 accessions from 7 species of AA genome in Oryza as donors. To date, more than 2000 SSSLs were developed, in which a rich of genes from AA genome of the Oryza genus was collected (He et al. 2005; Xi et al. 2006; He et al. 2017; Zhao et al. 2019). At the second step, we detected the genotypes on the chromosomal substituted segments in the SSSLs by mapping of genes or QTLs of important traits (He et al. 2005; Zhao et al. 2007; Liu et al. 2010; Zhang et al. 2012; Naeem et al. 2013; Yang et al. 2016; Zhou et al. 2017; Zhu et al. 2018), by assessing allelic variations at the loci of interest (Zeng et al. 2006; Teng et al. 2012; Cai et al. 2013), by analyzing interaction of gene by gene and gene by environment (Liu et al. 2008; Liu et al. 2012; Chen et al. 2014; Qin et al. 2015; Zhao et al. 2016; Yang et al. 2018), and by cloning genes of agronomic important (Wang et al. 2012; Wang et al. 2015). A large gene information has been collected from the SSSL library. At the third step, we conducted breeding by design using favorable genes selected in the library. For example, we have developed two varieties Huaxiaohei 1 and Huabiao 1, two CMS lines H121A and H131A, and two restorer lines H121R and H131R (Dai et al. 2015; Dai et al. 2016). In this study, we developed two new CMS lines HBA and HZA, four isonuclear alloplasmic CMS lines WA-HZA1, WAHZA2, DA-HZA, and YA-HZA, and two CMS lines with early heading and good quality, E5-HBA and E5HXA, on the SSSL platform. These results indicate that the SSSL library is a powerful platform for breeding by design in rice. Based on the gene information collected from the SSSL library, a series of varieties or lines could be designed. Then, the design could be achieved by pyramiding of the target genes in the SSSLs with the HJX74 genetic background.

In hybrid rice production, one of the key characteristics of CMS lines is of complete and steady sterility. In some CMS lines, the fertility can be easily influenced by the environmental factors. It was found that, for example, the individuals carrying the same genotypes in the same population from the WA-type indica CMS lines LongtefuA and BobaiA exhibited different fertility levels in different environmental conditions (Huang 1997; Lin and Cai 2002). In this study, we developed two CMS lines with early heading and good quality in the E5 genotype, E5-HXA and E5-HBA. The CMS in the E5-HXA line carrying the $r f 3$ and $r f 4$ genes from XieqingzaoA was complete and steady in different cropping seasons. However, the CMS in the E5-HBA line with the $r f 3$ and $r f 4$ genes of BobaiA was complete and steady in the fall cropping seasons, while incomplete and unsteady in the spring cropping seasons. It implies that the CMS in the different CMS lines carrying different $r f 3$ and $r f 4$ gene sources was in different fertility levels. We also found that the restored ability of E5-

Table 3 Pollen fertility of E5-HXA and E5-HBA on six sowing dates in 2015

\begin{tabular}{|c|c|c|c|c|c|c|}
\hline \multirow[t]{2}{*}{ CMS } & \multicolumn{6}{|c|}{ Pollen fertility (\%) } \\
\hline & Sowing on $3 / 2$ & Sowing on $3 / 18$ & Sowing on $4 / 1$ & Sowing on $5 / 1$ & Sowing on $6 / 1$ & Sowing on $7 / 13$ \\
\hline E5-HBA1 & $5.70 \pm 1.87$ & $1.11 \pm 1.11$ & - & 0 & 0 & 0 \\
\hline E5-HBA2 & $5.03 \pm 1.04$ & $1.42 \pm 0.66$ & $0.65 \pm 0.40$ & $0.12 \pm 0.09$ & 0 & 0 \\
\hline E5-HXA1 & 0 & 0 & 0 & 0 & 0 & 0 \\
\hline E5-HXA2 & 0 & 0 & 0 & 0 & 0 & 0 \\
\hline
\end{tabular}

Data shown are mean $\pm \mathrm{SD}, n=10$ 
HBA was better than that of E5-HXA in terms of the pollen fertility of the $\mathrm{F}_{1}$ hybrids derived from test crosses. These results indicated that in CMS lines, the higher in sterility level, the higher in abortive degree of pollens, the higher in sterility steadiness, but the lower in restored ability for CMS. The sterility and its restored ability in CMS lines depend on the $r f 3$ and $r f 4$ gene sources. Therefore, when designing CMS lines using $r f 3$ and $r f 4$ gene resources in the SSSL library, the balance between sterility and its restored ability in the lines should be concerned.

Author Contributions X.L. performed most of the experiments and prepared the data and the drafting of the manuscript; Z.D., W.Y., Q.T., Q.L., and J.G. performed some of the experiments; H.Z. performed the field experiments; G.L. performed the biometry; S.W. supervised this study and revised the manuscript; and G.Z. designed the experiments, supervised this study, and wrote the manuscript. All the authors have discussed the results and contributed to the drafting of the manuscript.

Funding information This work was supported by grants from the program of science and technology of Guangzhou (201607020040), from the National Natural Science Foundation of China (91735304 and 91435207), from the major program of transgenic new variety breeding of China (2014ZX08009-037B), and from the National Key Research and Development Program of China (2016YFD0100406).

\section{Compliance with ethical standards}

Conflict of interest The authors declare that they have no conflict of interest.

Open Access This article is distributed under the terms of the Creative Commons Attribution 4.0 International License (http:// creativecommons.org/licenses/by/4.0/), which permits unrestricted use, distribution, and reproduction in any medium, provided you give appropriate credit to the original author(s) and the source, provide a link to the Creative Commons license, and indicate if changes were made.

\section{References}

Cai J, Liao QP, Dai ZJ, Zhu HT, Zeng RZ, Zhang ZM, Zhang GQ (2013) Allelic differentiations and effects of the $R f 3$ and $R f 4$ genes on fertility restoration in rice with wild abortive cytoplasmic male sterility. Biol Plantarum 57(2):274-280

Chen X, Temnykh S, Xu Y, Cho YG, McCouch SR (1997) Development of a microsatellite framework map providing genome-wide coverage in rice (Oryza sativa L.). Theor Appl Genet 95:553-567
Chen JB, Li XY, Cheng C, Wang YH, Qin M, Zhu HT, Zeng RZ, $\mathrm{Fu}$ XL, Liu ZQ, Zhang GQ (2014) Characterization of epistatic interaction of QTLs $L H 8$ and $E H 3$ controlling heading date in rice. Sci Rep 4:4263

Cheng SH, Zhuang JY, Fan YY, Du JH, Cao LY (2007) Progress in research and development on hybrid rice: a superdomesticate in China. Ann Bot 100:959-966

Dai ZJ, Lu Q, Luan X, Cai J, Zhu HT, Liu ZQ, Zeng RZ, Zhang ZM, Wang SK, Zheng LJ, Li JL, Zhang GQ (2015) Development of a platform for breeding by design of CMS lines based on an SSSL library in rice (Oryza sativa L.). Euphytica 205:63-72

Dai ZJ, Lu Q, Luan X, Ouyang L, Guo J, Liang JY, Zhu HT, Wang WJ, Wang SK, Zeng RZ, Liu ZQ, Zhang ZM, Zhu XY, Zhang GQ (2016) Development of a platform for breeding by design of CMS restorer lines based on an SSSL library in rice (Oryza sativa L.). Breeding Sci 66:768-775

He FH, Xi ZY, Zeng RZ, Talukdar A, Zhang GQ (2005) Developing single segment substitution lines (SSSLs) in rice (Oryza sativa L.) using advanced backcrosses and MAS. Acta Genet Sin 32:825-831

He N, Wu RX, Pan XP, Peng LP, Sun K, Zou T, Zhu HT, Zeng RZ, Liu ZQ, Liu GF, Wang SK, Zhang GQ, Fu XL (2017) Development and trait evaluation of chromosome singlesegment substitution lines of $O$. meridionalis in the background of O. sativa. Euphytica 213:281-296

Huang RH (1997) Effect of different environmental factors on male-fertility stability of Longtepu A CMS lines. Fujian Sci Technol Rice Wheat 15(4):13-18

Kazama T, Toriyama K (2014) A fertility restorer gene, Rf4, widely used for hybrid rice breeding encodes a pentatricopeptide repeat protein. Rice 7:28

Li SQ, Yang DC, Zhu YG (2007) Characterization and use of male sterility in hybrid rice breeding. J Integr Plant Biol 49(6): 791-804

Lin JQ, Cai WJ (2002) Purification of a CMS line of hybrid rice BoA. Guangdong Agr Sci 3:9-10

Liu GF, Zhang ZM, Zhu HT, Zhao FM, Ding XH, Zeng RZ, Li WT, Zhang GQ (2008) Detection of QTLs with additive effects and additive-by-environment interaction effects on panicle number in rice (Oryza sativa L.) with singlesegment substitution lines. Theor Appl Genet 116:923-931

Liu GF, Zhu HT, Liu SW, Zeng RZ, Zhang ZM, Li WT, Ding XH, Zhao FM, Zhang GQ (2010) Unconditional and conditional QTL mapping for the developmental behavior of tiller number in rice (Oryza sativa L.). Genetica 138:885-893

Liu GF, Zhu HT, Zhang GQ, Li LH, Ye GY (2012) Dynamic analysis of QTLs on tiller number in rice (Oryza sativa L.) with single segment substitution lines. Theor Appl Genet 125:143-153

Luo DP, Xu H, Liu ZL, Guo JX, Li HY, Chen LT, Fang C, Zhang QY, Bai M, Yao N, Wu H, Wu H, Ji CH, Zheng HQ, Chen YL, Ye S, Li XY, Zhao XC, Li RQ, Liu YG (2013) A detrimental mitochondrial-nuclear interaction causes cytoplasmic male sterility in rice. Nat Genet 45:573-577

McCouch SR, Teytelman L, Xu YB, Lobos KB, Clare K, Walton M, Fu BY, Maghirang R, Li ZK, Xing YZ, Zhang QF, Kono I, Yano M, Fjellstrom R, DeClerck G, Schneider D, Cartinhour S, Ware D, Stein L (2002) Development and mapping of 2240 new SSR markers for rice (Oryza sativa L). DNA Res 6:199-207 
Naeem M, Freed S, Zhang GQ (2013) Molecular genetic studies of heading date gene OsMADS50 by using single segment substitution lines in Oryza sativa. Int J Agric Biol 15:631-639

Peleman JD, van der Voort JR (2003) Breeding by design. Trends Plant Sci 8:330-334

Qin M, Zhao XQ, Ru J, Zhang GQ, Ye GY (2015) Bigenic epistasis between QTLs for heading date in rice analyzed using single segment substitution lines. Field Crop Res 178:16-25

Sood BC, Siddiq EA (1978) A rapid technique for scent determination in rice. Indian J Genet Plant Breed 38(2):268-275

Spielmeyer W, Ellis MH, Chandler PM (2002) Semidwarf (sd-1), "green revolution" rice, contains a defective gibberellin 20oxidase gene. Proc Natl Acad Sci U S A 99:9043-9048

Tang HW, Luo DP, Zhou DG, Zhang QY, Tian DS, Zheng XM, Chen LT, Liu YG (2014) The rice restorer $R f 4$ for wildabortive cytoplasmic male sterility encodes a mitochondrial-localized PPR protein that functions in reduction of WA352 transcripts. Mol Plant 7:1497-1500

Teng B, Zeng RZ, Wang YC, Liu ZQ, Zhang ZM, Zhu HT, Ding XH, Li WT, Zhang GQ (2012) Detection of allelic variation at the $W x$ locus with single-segment substitution lines in rice (Oryza sativa L.). Mol Breeding 30:583-595

Teng B, Zhang Y, Wu JD, Cong XH, Wang RY, Han YH, Luo ZX (2013) Association between allelic variation at the Waxy locus and starch physicochemical properties using singlesegment substitution lines in rice (Oryza sativa L.). Starch 65:1-9

Wang SK, Wu K, Yuan QB, Liu XY, Liu ZB, Lin XY, Zeng RZ, Zhu HT, Dong GJ, Qian Q, Zhang GQ, Fu XD (2012) Control of grain size, shape and quality by OSSPL16 in rice. Nat Genet 44:950-954

Wang SK, Li S, Liu Q, Wu K, Zhang JQ, Wang SS, Wang Y, Chen XB, Zhang Y, Gao CX, Wang F, Huang HX, Fu XD (2015) The OSSPL16-GW7 regulatory module determines grain shape and simultaneously improves rice yield and grain quality. Nat Genet 47:949-954

Xi ZY, He FH, Zeng RZ, Zhang ZM, Ding XH, Li WT, Zhang GQ (2006) Development of a wide population of chromosome single-segment substitution lines in the genetic background of an elite cultivar of rice (Oryza sativa L.). Genome 49:476-484

Yang TF, Zhang SH, Zhao JL, Liu Q, Huang ZH, Mao XX, Dong JF, Wang XF, Zhang GQ, Liu B (2016) Identification and pyramiding of QTLs for cold tolerance at the bud bursting and the seedling stages by use of single segment substitution lines in rice (Oryza sativa L.). Mol Breeding 36:96

Yang ZF, Jin LL, Zhu HT, Wang SK, Zhang GQ, Liu GF (2018) Analysis of epistasis among QTLs on heading date based on single segment substitution lines in rice. Sci Rep 8:3059

Yao FY, Xu CG, Yu SB, Li JX, Gao YJ, Li XH, Zhang QF (1997) Mapping and genetic analysis of two fertility restorer loci in the wild-abortive cytoplasmic male sterility system of rice (Oryza sativa L.). Euphytica 98:183-187
Yuan LP (2017) Progress in super-hybrid rice breeding. Crop J 5: $100-102$

Zeng RZ, Zhang ZM, He FH, Xi ZY, Talukdar A, Shi JQ, Qin LJ, Huang CF, Zhang GQ (2006) Identification of multiple alleles at the $W x$ locus and development of single segment substitution lines for the alleles in rice. Rice Sci 13(1):9-14

Zhang GQ, Bharaj TS, Lu Y, Virmani SS, Huang N (1997) Mapping of the $R f-3$ nuclear fertility-restoring gene for WA cytoplasmic male sterility in rice using RAPD and RFLP markers. Theor Appl Genet 94:27-33

Zhang QY, Liu YG, Zhang GQ, Mei MT (2002) Molecular mapping of the fertility restorer gene $R f-4$ for WA cytoplasmic male sterility in rice. Acta Genet Sin 29:1001-1004

Zhang GQ, Zeng RZ, Zhang ZM, Ding XH, Li WT, Liu GF, He FH, Tulukdar A, Huang CF, Xi ZY, Qin LJ, Shi JQ, Zhao FM, Feng MJ, Shan ZL, Chen L, Guo XQ, Zhu HT, Lu YG (2004) The construction of a library of single segment substitution lines in rice (Oryza sativa L.). Rice Genet Newsl 21: $85-87$

Zhang ZH, Deng YJ, Tan J, Hu SN, Yu J, Xue QZ (2007) A genome-wide microsatellite polymorphism database for the indica and japonica rice. DNA Res 14:37-45

Zhang YX, Yang JY, Shan ZL, Chen S, Qiao WH, Zhu XY, Xie QJ, Zhu HT, Zhang ZM, Zeng RZ, Ding XH, Zhang GQ (2012) Substitution mapping of QTLs for blast resistance with SSSLs in rice (Oryza sativa L.). Euphytica 184:141-150

Zhao FM, Zhu HT, Ding XH, Zeng RZ, Zhang ZM, Li WT, Zhang GQ (2007) Detection of QTLs for important agronomic traits and analysis of their stabilities using SSSLs in rice. Agr Sci China 6:769-778

Zhao FM, Zhu HT, Zeng RZ, Zhang GQ, Xu SZ (2016) Detection of additive and additive $\times$ environment interaction effects of QTLs for yield-component traits of rice using single-segment substitution lines (SSSLs). Plant Breed 135:452-458

Zhao HW, Sun LL, Xiong TY, Wang ZQ, Liao Y, Zou T, Zheng MM, Zhang Z, Pan XP, He N, Zhang GQ, Zhu HT, Liu ZQ, He P, Fu XL (2019) Genetic characterization of the chromosome single-segment substitution lines of $O$. glumaepatula and $O$. barthii and identification of QTLs for yield-related traits. Mol Breeding 39:51

Zhou YL, Xie YH, Cai JL, Liu CB, Zhu HT, Jiang R, Zhong YY, Zhang GL, Tan B, Liu GF, Fu XL, Liu ZQ, Wang SK, Zhang GQ, Zeng RZ (2017) Substitution mapping of QTLs controlling seed dormancy using single segment substitution lines derived from multiple cultivated rice donors in seven cropping seasons. Theor Appl Genet 130:1191-1205

Zhu HT, Li Y, Liang JY, Luan X, Xu P, Wang SK, Zhang GQ, Liu GF (2018) Analysis of QTLs on heading date based on single segment substitution lines in rice (Oryza sativa L.). Sci Rep $8: 13232$

Publisher's note Springer Nature remains neutral with regard to jurisdictional claims in published maps and institutional affiliations. 